\title{
Formação humana, pesquisa e produção científica na subárea de avaliação "nutrição" da Coordenação de Aperfeiçoamento de Pessoal de Nível Superior, no Brasil, de 2007 a 2009
}

\section{Human resources, research and scientific production \\ of Brazilian Graduate Programs in}

Nutrition, 2007-2009

Maria Teresa Anselmo OLINTO ${ }^{1,5}$

Pedro Israel Cabral de LIRA²

Julio Sergio MARCHINI ${ }^{3}$

Gilberto KAC ${ }^{4}$

RES U M O

\section{Objetivo}

Descrever o perfil da Pós-Graduação stricto sensu na subárea "Nutrição" da Coordenação de Aperfeiçoamento de Pessoal de Nível Superior, para o triênio 2007-2009, no Brasil.

\section{Métodos}

Os dados foram extraídos do sítio da Coordenação de Aperfeiçoamento de Pessoal de Nível Superior. Foram analisados 17 programas para os indicadores: número de alunos novos, número total de orientações concluídas, tempo mediano de titulação, número total e média de docentes permanentes por programa. A produção científica foi classificada segundo o Qualis-Capes disponível para o triênio 2007-2009. Foram listadas as 35 revistas com o maior número de artigos publicados por professores da subárea.

\section{Resultados}

No período houve ingresso de 618 alunos de mestrado e 241 de doutorado. Foram concluídas 596 dissertações e 178 teses. O número de docentes permanentes passou de 163 em 2007 para 224 em 2009, aumentando 37,4\%. Foram publicados 2168 artigos, 10,9\% em periódicos A1 e A2, 48,8\% em periódicos B1 a B3 e $40,3 \%$ em periódicos B4 e B5. Os periódicos British Journal of Nutrition ( $n=12$, nível A2), Food Chemistry

\footnotetext{
${ }^{1}$ Universidade do Vale do Rio dos Sinos, Programa de Pós-Graduação em Saúde Coletiva. Av. Unisinos, 950, 93022-000, São Leopoldo, RS, Brasil. Correspondência para/Correspondence to: M.T.A. OLINTO. E-mail: <mtolinto@unisinos.br>.

2 Universidade Federal de Pernambuco, Programa de Pós-Graduação em Nutrição. Recife, PE, Brasil.

${ }^{3}$ Universidade de São Paulo, Faculdade de Medicina, Departamento de Clínica Médica. Ribeirão Preto, SP, Brasil.

${ }^{4}$ Universidade Federal do Rio de Janeiro, Instituto de Nutrição Josué de Castro, Observatório de Epidemiologia Nutricional. Rio de Janeiro, RJ, Brasil.

${ }^{5}$ Universidade Federal de Ciência da Saúde de Porto Alegre, Departamento de Nutrição. Porto Alegre, RS, Brasil.
} 
918 | M.T.A. OLINTO et al.

( $n=12$, nível A2), Journal of Agricultural and Food Chemistry ( $n=11$, nível A2), Revista de Nutrição ( $n=100$, nível B4), Cadernos de Saúde Pública ( $n=63$, nível B3) e Ciência e Saúde Coletiva $(n=63$, nível B3) foram os que apresentaram o maior número de artigos publicados por professores dos programas analisados em revistas internacionais e nacionais, respectivamente.

\section{Conclusão}

O crescimento dos programas em questão foi notável, sobretudo no número de alunos titulados e professores permanentes. Há necessidade de um incremento no perfil de publicação no futuro. Espera-se importante impacto nos indicadores de avaliação após a criação da área de "Nutrição" na Coordenação de Aperfeiçoamento de Pessoal de Nível Superior.

Termos de indexação: Alimentação. Nutrição. Programa de pós-graduação. Pesquisa.

\section{A B S T R A C T}

\section{Objective}

This study described the profile of graduate nutrition programs for the years 2007-2009 in Brazil.

\section{Methods}

Data was obtained from the Coordenação de Aperfeiçoamento de Pessoal de Nível Superior (Coordination for the Improvement of Higher Education Personnel) website. The following indicators were used to analyze 17 programs: number of newly enrolled students, total number of completed supervisions, median time to graduate, and total and average number of tenured supervisors per program. Research papers were analyzed according to the Qualis-Coordenação de Aperfeiçoamento de Pessoal de Nível Superior criteria available for the years 2007-2009. The 35 journals with the highest number of articles published by supervisors in the area were listed.

\section{Results}

During the period there were 618 masters and 241 doctoral students. A total of 596 dissertations and 178 theses had been completed. The number of tenured supervisors increased from 163 in 2007 to 224 in 2009, an increase of $37.4 \%$. A total of 2,168 research articles were published, $10.9 \%$ in journals $A 1$ and $A 2,48.8 \%$ in B1 to B3, and $40.3 \%$ in B4 and B5. The British Journal of Nutrition ( $n=12$, level A2), Food Chemistry $(n=12$, level A2), Journal of Agricultural and Food Chemistry $(n=11$, level A2), Revista de Nutrição ( $n=100$, level B4), Cadernos de Saúde Pública ( $n=63$, level B3) and Ciência e Saúde Coletiva ( $n=63$, level B3) had the largest number of articles published by supervisors in the programs analyzed for international and national journals, respectively.

\section{Conclusion}

The growth of nutrition programs was notable, especially regarding the number of graduates and tenured supervisors. There is room for improvement in publication profile.

Indexing terms: Feeding. Nutrition. Graduate program. Research.

\section{N T R O D U Ç Ã O}

No Brasil, os programas de Pós-Graduação stricto sensu (mestrado e doutorado) recomendados e reconhecidos pela Coordenação de Aperfeiçoamento de Pessoal de Nível Superior (CAPES) fornecem informações que compõem relatórios utilizados no processo de avaliação e que dão origem aos Cadernos de Indicadores. Esses Cadernos contêm dados qualitativos e quantitativos sobre a proposta do programa, as linhas e projetos de pesquisa, o corpo docente com vínculo de formação, as disciplinas, as teses e dissertações, bem como a produção científica docente e discente. O conjunto dessas informações encontrase disponível para consulta pública (www.capes. gov.br/avaliacao), o que permite o acompanhamento do desenvolvimento da Pós-Graduação stricto sensu no País.

Ao final da avaliação trienal (2007-2009), ocorrida em agosto de 2010, o universo da Pós-Graduação (stricto sensu) era composto por 2928 programas e 4394 cursos de mestrado 
acadêmico, mestrado profissional e doutorado. Desse total, 484 programas e 772 cursos estavam inseridos na grande área das Ciências da Saúde, o que faz desta a maior entre as nove grandes áreas que conformam o Sistema de Pós-Graduação da Capes. A grande área da saúde corresponde a $17 \%$ do total de programas (Capes, 2010). No interior da grande área das Ciências da Saúde havia oito áreas de avaliação: Enfermagem; Educação Física, Fonoaudiologia, Fisioterapia e Terapia Ocupacional; Farmácia; Odontologia; Saúde Coletiva e as Medicinas, divididas em três: I, II e III.

No início de 2010, a Medicina II, era a maior área de avaliação, com 91 programas (19\% da Grande Área) e 159 cursos; incluía-se aí a subárea de Nutrição, representada por 17 programas e 25 cursos, no triênio de 2007-2009.

Uma vez que esses programas não se constituíam em uma área específica na grande área da Saúde e seus cursos permaneciam vinculados à área da Medicina II, desde 2006, um movimento liderado pelo Fórum Nacional de Coordenadores de Programas de Pós-Graduação em Alimentação e Nutrição (Fórum PPG A\&N) passou a fazer gestões junto à Capes com vistas à criação de uma área específica ${ }^{1-3}$. Registre-se, por oportuno, que Alimentação e Nutrição (A\&N), como campo científico, corresponde a espaço social de grande amplitude e complexidade, encontrando expressão em diversos outros lugares do mundo da produção de conhecimentos e saberes, bastante além das áreas e subáreas da Capes e demais instituiç̧ões correlatas ${ }^{4}$.

As oportunidades de formação pós-graduada em A\&N têm aumentado de forma importante nos últimos anos no Brasil, mas não existe um mapeamento geral recente desses programas. O presente artigo busca superar essa lacuna, tendo como objetivo descrever o perfil da formação humana, da pesquisa e da produção científica no âmbito dos programas de Pós-Graduação stricto sensu inseridos na subárea de Nutrição, avaliada pela Capes, no triênio 2007-2009 no Brasil.

\section{MÉ T O D O S}

Foram utilizados os dados fornecidos a Capes pelos programas específicos da subárea de Nutrição. Para cada programa foram consultadas as informações presentes nos Cadernos de Indicadores referentes a corpo docente, corpo discente, linhas e projetos de pesquisa e produção científica, no triênio 2007-2009.

Foram avaliados os seguintes indicadores para cada um dos anos do triênio: número de alunos novos, número total de orientações concluídas, tempo mediano de titulação, número total de docentes permanentes, média de docentes permanentes por programa, proporção de docentes colaboradores e número total de projetos de pesquisa em andamento. Os três primeiros indicadores foram avaliados separadamente para o mestrado e doutorado. Para a definição da área de atuação dos docentes, foram utilizadas as informações referentes a titulação pós-graduada - nível doutorado.

Para avaliar a produção científica dos programas no formato de artigos, elaborou-se uma lista com os 35 periódicos nos quais os professores dos programas em tela mais publicaram no triênio. Essa lista foi dividida segundo o estrato Qualis- Capes adotado pela área de Medicina II. Foram listados 15 periódicos classificados como Qualis A1 ou A2, e 20 classificados como Qualis B1, B2, B3, B4 ou B5. Além do nível Qualis da Capes, foi apresentado o fator de impacto medido pela Thompson-Reuters e publicado anualmente no Journal of Citation Report (JCR). O JCR empregado na avaliação trienal referiu-se ao ano de 2008 (http://qualis.capes.gov.br/webqualis/).

A análise contemplou ainda a apresentação da distribuição absoluta e relativa do número total de artigos publicados pelo conjunto dos programas segundo o estrato Qualis-Capes. A distribuição relativa foi apresentada com e sem os dados do programa da Universidade Federal do Rio Grande do Norte (UFRN), por se tratar de um programa multidisciplinar. 
A coleta de dados ocorreu em dois momentos julho e outubro de 2010, após a divulgação dos resultados da avaliação trienal da Capes. Por serem todas informações de cunho público, não houve necessidade de aprovação pelo comitê de ética em pesquisa. Os resultados contidos neste artigo não apresentam caráter classificatório dos programas dentro da subárea, referindo-se a informações descritivas que poderão ser utilizadas como linha de base para comparação em avaliações futuras.

\section{RESULTADOS}

Ao final do triênio de 2007-2009, a subárea de Nutrição estava constituída por 17 programas, inseridos em 16 universidades públicas, das quais 14 federais e 2 estaduais, distribuídas nas seguintes unidades federativas: Paraíba $(n=1)$, Pernambuco ( $n=2)$, Rio Grande do Norte $(n=1)$, Alagoas $(n=1)$, Bahia $(n=1)$, Mato Grosso $(n=1)$, Goiás $(n=1)$, Minas Gerais ( $n=2)$, Distrito Federal $(n=1)$, Rio de Janeiro ( $n=2)$, São Paulo $(n=2)$, Santa Catarina $(n=1)$ e Rio Grande do Sul $(n=1)$. A região
Nordeste apresentava o maior número de programas ( $n=6)$, e a Sul, o menor ( $n=2)$. Observa-se que $70 \%$ dos programas em funcionamento tiveram sua estrutura atual implantada no início do anos 2000. Dois programas foram implantados somente no início de 2010, o da Universidade Federal de Pelotas (UFPel) e o da Universidade Federal de Ouro Preto (UFOP). Dos 17 programas, 8 tinham cursos de doutorado (Tabela 1). Nesta Tabela também é possível observar-se o número de linhas de pesquisa em cada programa ao final do triênio, no ano de 2009. Em média, observam-se quatro linhas de pesquisa/programa (mediana=3), destacando-se as universidades federais de Pernambuco e do Rio Grande do Norte, com 12 e 10 linhas de pesquisa, respectivamente.

As informações dos Cadernos de Indicadores listavam, no sítio da Capes, 11 programas em 2007, e 15 em 2009. No período 2007-2009, houve ingresso de 618 alunos de mestrado e 241 de doutorado, bem como a conclusão de 596 dissertações e 178 teses. As defesas de doutorado ocorreram em quatro Programas - UFRN, Universi-

Tabela 1. Programas de pós-graduação (stricto sensu) da área de nutrição, vínculo institucional, período de implantação e nível do curso, ano 2010.

\begin{tabular}{|c|c|c|c|c|c|}
\hline & \multirow{2}{*}{ Programa' } & \multirow{2}{*}{ Instituição de Ensino Superior } & \multicolumn{2}{|c|}{ Início } & \multirow{2}{*}{ - Linhas de Pesquisa } \\
\hline & & & Mestrado & Doutorado & \\
\hline 1 & Nutrição & Universidade Federal de Pernambuco - UFPE & 1971 & 1991 & 12 \\
\hline 2 & Nutrição & Universidade Federal do Rio de Janeiro - UFRJ & 1985 & 2006 & 4 \\
\hline 3 & Nutrição Humana e Aplicada & Universidade de São Paulo - USP & 1991 & 1991 & 3 \\
\hline 4 & Nutrição & Universidade Federal de São Paulo - UNIFESP & 1991 & 1991 & 4 \\
\hline 5 & Ciências da Nutrição & Universidade Federal da Paraíba - UFPb & 1995 & - & 3 \\
\hline 6 & Nutrição Humana & Universidade de Brasília - UNB & 2000 & 2009 & 4 \\
\hline 7 & Ciências da Saúde & Univ. Federal do Rio Grande do Norte - UFRN & 2001 & 2005 & 10 \\
\hline 8 & Ciência da Nutrição & Universidade Federal de Viçosa - UFV & 2001 & 2009 & 2 \\
\hline 9 & Nutrição & Universidade Federal de Santa Catarina - UFSC & 2002 & - & 2 \\
\hline 10 & Alimentos, Nutrição e Saúde & Universidade Federal da Bahia - UFBA & 2005 & - & 5 \\
\hline 11 & Nutrição & Universidade Federal de Alagoas -UFAL & 2005 & - & 3 \\
\hline 12 & Alimentação, Nutrição e Saúde & Universidade do Estado do Rio de Janeiro - UERJ & 2008 & 2010 & 3 \\
\hline 13 & Biociências & Universidade Federal do Mato Grosso - UFMT & 2008 & - & 3 \\
\hline 14 & Saúde Humana e Meio Ambiente ${ }^{2}$ & Universidade Federal de Pernambuco - UFPE & 2009 & - & 5 \\
\hline 15 & Nutrição e Saúde & Universidade Federal de Goiás - UFG & 2009 & - & 3 \\
\hline 16 & Nutrição e Alimentos & Universidade Federal de Pelotas - UFPel & 2010 & - & 2 \\
\hline 17 & Saúde e Nutrição & Universidade Federal de Ouro Preto - UFOP & 2010 & - & 2 \\
\hline
\end{tabular}

${ }^{1}$ Programas listados em ordem de implantação do mestrado; ${ }^{2}$ Campus avançado de Vitória de Santo Antão.

Fonte: Cadernos de Avaliação da Capes ${ }^{4}$. 
dade de São Paulo, Universidade Federal de São Paulo e Universidade Federal de Pernambuco, estando $40 \%$ delas concentradas na primeira universidade - o que, ressalte-se, é um valor superestimado em razão de ser este um programa multidisciplinar, com formação humana em outros campos além de Alimentação e Nutrição. Nesse sentido, excluídos os valores correspondentes ao Programa da UFRN, observou-se que na subárea de Nutrição houve o ingresso de 94 alunos no doutorado e a conclusão de 98 teses, no período 2007-2009. O número total de docentes permanentes aumentou $37,4 \%$, passando de 163 , em 2007, para 224 em 2009. Considerando-se todos os programas, o número médio de docentes permanentes máximo foi de 16, e o mínimo, de 13. Observou-se redução da proporção de docentes colaboradores ao longo do triênio, passando de 46\% para 33\% (Tabela 2).

No triênio, foi publicado um total de 2168 artigos com classificação Qualis-Capes, excluindo-se as publicações do programa com perfil multidisciplinar. Dentre esses artigos, 237 foram publicados em periódicos classificados como A1 e A2,
1058 em periódicos B1 a B3, e 873 em periódicos B4 a B5. A Figura 1 mostra a distribuição proporcional da publicação com e sem a contabilização do Programa da UFRN, no qual parte da produção científica não é produzida no âmbito do campo alimentar e nutricional. Observa-se que não há muita diferença nos percentuais, referentes à produção Qualis A1 e A2. A Figura 1 evidencia que a produção científica da subárea de Nutrição está concentrada entre os estratos B3 e B5.

A Tabela 3 apresenta a lista dos 15 periódicos classificados como Qualis-Capes A1 e A2 com maior número de artigos publicados oriundos dos programas, e o fator de impacto relativo ao JCR de 2008. Os periódicos British Journal of Nutrition ( $n=12)$, Food Chemistry $(n=12)$ e Journal of Agricultural and Food Chemistry $(n=11)$ foram aqueles com maior número de artigos. Os periódicos Clinical Nutrition ( $\mathrm{n}=9$ ), Obesity Surgery $(\mathrm{n}=9)$; Hormone and Metabolic Research $(n=6)$ e Life Sciences $(n=6)$ também apareceram de forma importante.

A Tabela 4 apresenta os 20 periódicos classificados como Qualis-Capes entre B1 e B5

Tabela 2. Indicadores acadêmicos e de pesquisa dos programas (stricto sensu) da área de nutrição, período 2007 a 2009.

\begin{tabular}{|c|c|c|c|}
\hline \multirow{3}{*}{ Indicadores } & \multicolumn{3}{|c|}{ Ano } \\
\hline & 2007 & 2008 & 2009 \\
\hline & $\mathrm{n}=11^{(1)}$ & $\mathrm{n}=11^{(1)}$ & $n=15^{(1)}$ \\
\hline Alunos novos & $190(154)$ & $174(130)$ & $254(242)$ \\
\hline Orientações concluídas & $201(185)$ & $203(180)$ & $192(169)$ \\
\hline Tempo mediano de titulação (meses) & \multicolumn{3}{|c|}{ Doutorado $^{2}$} \\
\hline Alunos novos & $89(34)$ & $99(29)$ & $53(31)$ \\
\hline Orientações concluídas & $54(32)$ & $58(33)$ & $66(33)$ \\
\hline Tempo mediano de titulação (meses) & $46(49)$ & $45(50)$ & $44(47)$ \\
\hline Média dos programas & $14,8(13,4)$ & $15,5(13,5)$ & $16,0(14)$ \\
\hline$\%$ docentes colaboradores/permanentes & $46(46)$ & $44(46)$ & $33(35)$ \\
\hline Projetos de Pesquisa em andamento & $699(601)$ & $728(559)$ & $940(784)$ \\
\hline
\end{tabular}

${ }^{1}$ Valores entre parênteses correspondem ao resultado do indicador após a exclusão do Programa da UFRN; ${ }^{2}$ Os indicadores referentes ao doutorado estão baseados em dados de cinco programas.

Fonte: Cadernos de Avaliação da Capes ${ }^{4}$. 
com maior número de publicações. Observa-se que a Revista de Nutrição foi aquela que apresentou o maior número de publicações provenientes dos programas da área $(n=100)$, seguida de dois periódicos com temática ligada à saúde coletiva: Cadernos de Saúde Pública $(n=63)$ e Ciência e Saúde Coletiva $(n=63)$, totalizando 126 artigos no triênio.

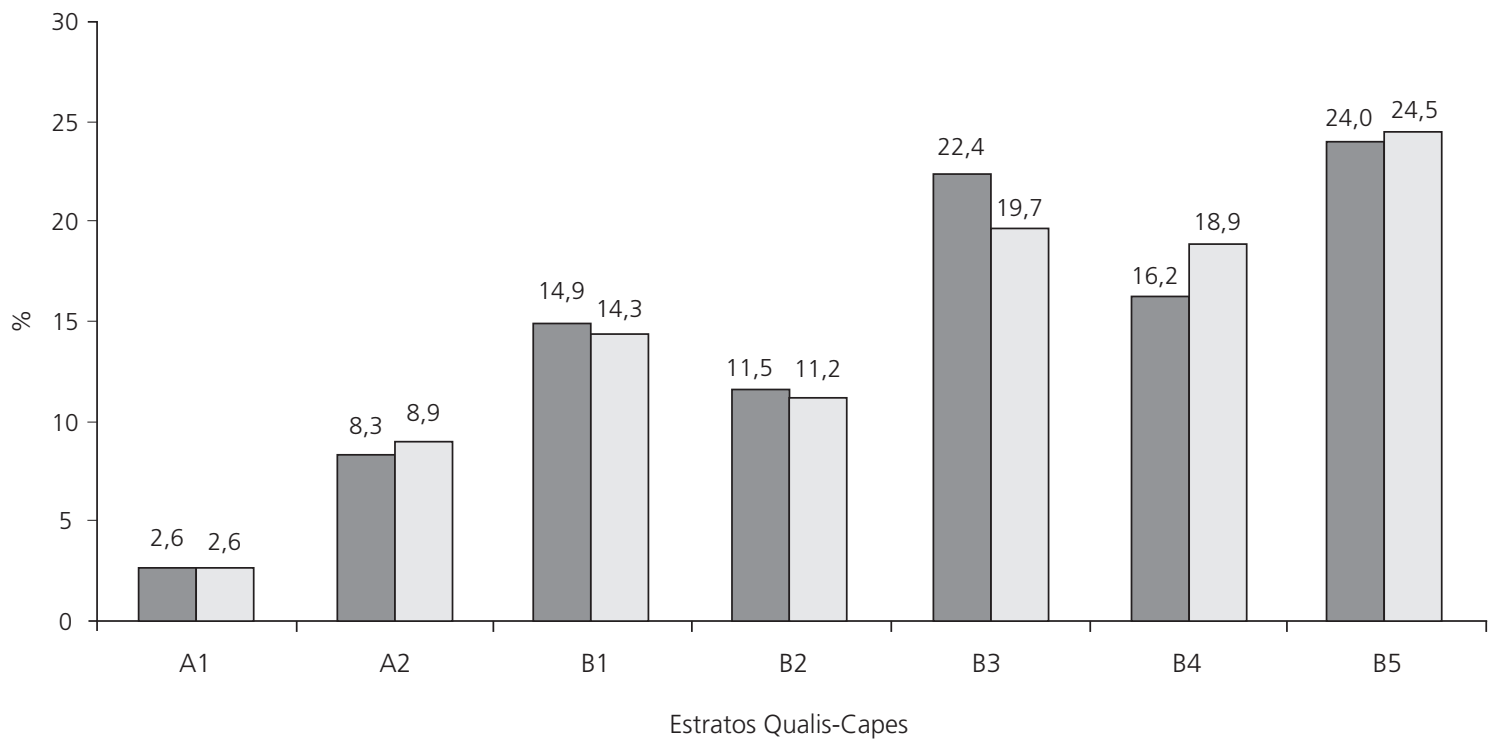

Figura 1. Proporção de artigos científicos publicados na área de nutrição, classificado segundo estratos Qualis-Capes de periódicos (A1 até B5), no período de 2007 a 2009.

Nota: Nas barras mais claras estão excluídos os artigos referentes a um programa com característica interdisciplinar

Fonte: Cadernos de Avaliação da Capes4.

Tabela 3. Lista dos 15 periódicos científicos com maior número de publicações provenientes dos programas de pós-graduação de nutrição, por estratos A1 e A2 Qualis-Capes de periódicos, período 2007 a 2009.

\begin{tabular}{llccc}
\hline \multirow{2}{*}{ Ordem $^{1}$} & \multicolumn{4}{c}{ Periódicos Qualis-Capes A1 e A2 } \\
\cline { 2 - 5 } & Nome do periódico & Qualis & Fator de Impacto (2008) & Artigos (n) \\
\hline $1^{\circ}$ & British Journal of Nutrition & A2 & 2,764 & 12 \\
$2^{\circ}$ & Food Chemistry & A2 & 2,696 & 12 \\
$3^{\circ}$ & Journal of Agricultural and Food Chemistry & A2 & 2,562 & 11 \\
$4^{\circ}$ & Clinical Nutrition & A2 & 3,203 & 9 \\
$5^{\circ}$ & Obesity Surgery & A2 & 2,913 & 9 \\
$6^{\circ}$ & Hormone and Metabolic Research & A2 & 2,715 & 6 \\
$7^{\circ}$ & Life Sciences & A2 & 2,583 & 6 \\
$8^{\circ}$ & Journal of Nutrition Biochemistry & A1 & 4,352 & 4 \\
$9^{\circ}$ & Journal of Food Composition and Analysis & A2 & 2,457 & 4 \\
$10^{\circ}$ & Experimental Neurology & A1 & 3,974 & 3 \\
$11^{\circ}$ & International Journal of Obesity & A2 & 3,640 & 3 \\
$12^{\circ}$ & International Journal of Cardiology & A2 & 3,121 & 3 \\
$13^{\circ}$ & Journal of Hazardous Materials & A2 & 2,975 & 3 \\
$14^{\circ}$ & American Journal of Kidney Diseases & A1 & 4,822 & 2 \\
$15^{\circ}$ & American Journal of Public Health & A1 & 4,241 & 2 \\
\hline
\end{tabular}

${ }^{1}$ Foram incluídos dados de 14 de 17 programas pois dois foram implantados em 2010 e um possui característica multidisciplinar, que poderia levar a pequena distorção no perfil das publicações.

Fonte: Cadernos de Avaliação da Capes . 
Tabela 4. Lista dos 20 periódicos científicos com maior número de publicações provenientes dos programas de pós-graduação de nutrição, estratos B1 a B5 Qualis-Capes de periódicos, período 2007 a 2009.

\begin{tabular}{|c|c|c|c|c|}
\hline Ordem ${ }^{1}$ & \multicolumn{4}{|c|}{ Periódicos Qualis-Capes B1 a B5 } \\
\hline $2^{\circ}$ & Cadernos de Saúde Pública & B3 & - & 63 \\
\hline $3^{\circ}$ & Ciência e Saúde Coletiva & B3 & - & 63 \\
\hline $4^{\circ}$ & Brazilian Journal of Medical and Biological Research & B1 & 1,215 & 41 \\
\hline $6^{\circ}$ & Nutrição em Pauta & B5 & - & 34 \\
\hline $7^{\circ}$ & Nutrire & B5 & - & 33 \\
\hline $8^{\circ}$ & Ciência e Tecnologia de Alimentos & B4 & - & 31 \\
\hline $9^{\circ}$ & Jornal de Pediatria & B3 & - & 31 \\
\hline $10^{\circ}$ & Revista Brasileira de Nutrição Clínica & B5 & - & 31 \\
\hline $14^{\circ}$ & Higiene Alimentar & B5 & - & 26 \\
\hline $15^{\circ}$ & Revista Brasileira de Medicina do Esporte & B4 & - & 25 \\
\hline $16^{\circ}$ & Nutrition & B1 & 2,280 & 24 \\
\hline $17^{\circ}$ & Revista de Saúde Pública & B2 & 0,963 & 20 \\
\hline $18^{\circ}$ & Archivos Latinoamericanos de Nutrición & B2 & 0,316 & 19 \\
\hline $19^{\circ}$ & Nutrición Hospitalaria & B2 & 1,096 & 14 \\
\hline $20^{\circ}$ & Arquivos Brasileiros de Cardiologia & B3 & - & 14 \\
\hline
\end{tabular}

${ }^{1}$ Foram incluídos dados de 14 de 17 programas visto que dois foram implantados em 2010 e outro programa apresenta características multidisciplinar, que poderia levar a pequena distorção no perfil das publicações.

Fonte: Cadernos de Avaliação da Capes ${ }^{4}$.

Foram identificadas 290 diferentes denominações para as linhas de pesquisa, 22\% das quais denominadas como Nutrição. Na sequência identificaram-se $13 \%$ das áreas vinculadas diretamente a saúde coletiva, e $8 \%$ vinculadas a ciências e tecnologia dos alimentos. O restante (57\%) apresentou-se disperso em diversos campos do conhecimento, como farmacologia, fisiologia, odontologia, biofísica, entre outros (resultados não apresentados em Tabelas).

\section{DISCUSS Ã O}

Os resultados do presente estudo revelaram diversos aspectos para reflexão acerca do perfil apresentado pelos programas, no triênio 2007-2009, com base nos indicadores contidos nos Cadernos de Indicadores da Capes. Entre os aspectos positivos deve-se destacar o impressionante crescimento no número de programas.
Seis dos atuais 17 programas foram criados entre 2007 e 2010, representando um crescimento de $35 \%$. Também foi considerável o aumento de $34 \%$ no número de alunos novos de mestrado, bem como o aumento de $37 \%$ no número de docentes permanentes e de $34 \%$ no número de projetos de pesquisa. Considerando os três anos do triênio, houve ingresso de 241 alunos no doutorado, valor considerado expressivo para uma área com apenas 8 cursos de doutorado. Destaca-se o número total de conclusões de teses de doutorado - 178 ao longo do triênio. A relação de concluintes entre mestrado e doutorado para a área foi de 3,3 para 1, valor levemente superior ao apontado para o país. Espera-se que esta relação se iguale à medida que sejam oferecidos mais cursos de doutorado na área. Outro aspecto positivo diz respeito à excelente distribuição geográfica que esses programas apresentam. Exceto pela ausência de programas na região Norte, há programas implantados em todas as 
regiões do país. Salienta-se que a grande maioria deles iniciou suas atividades nesta última década, ou seja, são considerados programas novos.

Os programas estavam compostos por 70 linhas de pesquisa, o que mostra a consolidação do campo do conhecimento da A\&N. Além disso, a qualidade da produção científica e da formação dos recursos humanos expressa o grau de maturidade da pesquisa.

Embora tenha havido considerável aumento qualitativo e quantitativo no número de publicações, é importante salientar que o conjunto de programas precisa melhorar ainda mais o seu perfil de publicação. Isso fica claro ao se observar que apenas 10,9\% dos artigos do triênio foram publicados em revistas Qualis-Capes A1 e A2. Outro dado não tão positivo diz respeito à proporção de artigos Qualis-Capes igual ou superior a B2, a qual deveria alcançar em torno de $50 \%$, mas que para o conjunto de programas de $\mathrm{A} \& \mathrm{~N}$ alcançou apenas 39,3\%. Deve-se salientar ainda o fato de que, das dez revistas Qualis-Capes entre B1 e B5 nas quais os professores desses programas mais publicaram, três apresentaram nível B5, totalizando 108 artigos, equivalentes a cerca de 5\% do total de artigos publicados.

Algumas limitações devem ser destacadas no que se refere à forma como o presente mapeamento foi realizado. A primeira delas diz respeito ao principal critério de inclusão no estudo: ser um programa stricto sensu e estar vinculado à subárea de Nutrição, alocada na área de Medicina II da Capes quando os dados analisados foram gerados. É necessário, ainda, assinalar a existência de programas que compõem o campo científico Alimentação e Nutrição inseridos em outras áreas de avaliação da Capes, que não a Medicina II, como a Saúde Coletiva e a Ciência de Alimentos, especialmente.

Por outro lado, salienta-se que um aspecto positivo dos dados constantes neste artigo diz respeito a sua publicização, considerando-se que os mesmos foram obtidos nas planilhas informadas oficialmente pelos Programas à Capes. Outro aspecto positivo é que esse mapeamento pode servir de linha de base para a comparação com dados futuros e para a definição de prioridades e necessidades na nova área de Nutrição, recentemente criada.

Um ponto de discussão importante a ser comentado, e que diz respeito aos resultados do presente artigo, refere-se ao efeito positivo do trabalho desenvolvido pelo Fórum PPG A\&N desde 2006. A organização do conjunto de programas em $\mathrm{A} \& \mathrm{~N}$, em uma área própria na Capes, consiste em conquista de grande importância. Desde 2006, o Fórum tem implementado atividades que visam ao fortalecimento técnico e político dos programas em $A \& N$ e teve na criação da área sua maior vitória ${ }^{5}$. A criação dessa área, recentemente aprovada pelo Comitê Técnico Cientifico do Ensino Superior da Capes, possibilitará conquistas mais permanentes, como, por exemplo, o estabelecimento de critérios de avaliação mais apropriados, a indução à criação de programas em regiões geográficas pouco privilegiadas do país, assim como o estabelecimento de metas e prioridades claras para o crescimento acadêmico da área.

Em resumo, conclui-se que o conjunto de programas progrediu de forma substancial no último triênio, a saber: 1) melhoria importante nos indicadores, tais como o número de alunos de mestrado e doutorado titulados; 2) ampliação do número de docentes permanentes; 3) expansão da área com novos programas; 4) fortalecimento político da área. Sabe-se que ainda há um grande desafio a ser enfrentado, sobretudo no que diz respeito à qualificação do conhecimento produzido no bojo desses programas.

Espera-se que a criação da área de Nutrição na Capes aglutine boa parte do conhecimento produzido e dê maior visibilidade ao campo científico Alimentação e Nutrição como um todo. Espera-se ainda que ela garanta o fomento mínimo advindo de editais da Capes distribuídos pela totalidade das áreas, bem como permita a criação de critérios próprios de avaliação, mais coadunados com a realidade da área. 


\section{REFER Ê N CIAS}

1. Kac G, Fialho E, Santos SMC, Assis AMO. Reflexões do I fórum de coordenadores de programas de pós-graduação em nutrição no Brasil. Rev Nutr. 2006; 19(6):785-92. doi: 10.1590/S1415-527320 06000600013.

2. Kac G, Fialho E, Santos SMC. Panorama atual dos programas de pós-graduação em Nutrição no Brasil. Rev Nutr. 2006; 19(6):771-84. doi: 10.1590/S 1415-52732006000600012.

3. Castro RM, Osório M, Leandro CG, Kac G. II Fórum de coordenadores de Programas de Pós-Graduação em Nutrição no Brasil. Rev Bras Saúde Matern
Infant. 2007; 7(3)235-36. doi: 10.1590/\$1519-38 292007000300001.

4. Coordenação de Aperfeiçoamento de Pessoal de Nível Superior. Relação de Cursos Recomendados e Reconhecidos. Atualização em 31 de agosto de 2010. Brasília; 2010 [acesso 2010 nov. 23]. Disponível em: <http://conteudoweb.capes.gov.br/ conteudoweb/ProjetoRelacaoCursos>.

5. Kac G, Proença RPC, Prado SD. A criação da área de Nutrição na CAPES. Rev Nutr. 2011; 24(6): 905-16.

\section{Recebido em: 19/9/2011}

Versão final reapresentada em: 14/11/2011

Aprovado em: 21/11/2011 
